\title{
Stiff Chain Behavior of Poly(phthaloyl-trans-2,5-dimethylpiperazine) in Dilute Solution
}

\author{
Masanori Motowoka, Takashi Norisuye, and Hiroshi Fujita \\ Department of Polymer Science, Osaka University, \\ Toyonaka, Osaka 560, Japan.
}

(Received May 16, 1977)

\begin{abstract}
Fractionated samples of poly(phthaloyl-trans-2,5-dimethylpiperazine) in various organic solvents at $25^{\circ} \mathrm{C}$ were studied by light scattering, viscosity, and sedimentation velocity. The data obtained for $z$-average mean-square radii of gyration $\left\langle S^{2}\right\rangle_{z}$, intrinsic viscosities [r], and sedimentation coefficients $s_{0}$ exhibited features predictable for stiff or semiflexible chains. Thus an attempt was made to analyze the data by combination of the Yamakawa-Fujii theory for intrinsic viscosity and the Benoit-Doty expression for mean-square radius of gyration of unperturbed wormlike chains with a reasonable assumption for the partial specific volume. In this way, three parameters $q, M_{\mathrm{L}}$, and $d$ characterizing the wormlike cylinder were estimated for each solvent; for example, $q=33 \AA, M_{\mathrm{L}}=33$ daltons $/ \AA$, and $d=7.4 \AA$ in $N$-methyl-2pyrrolidone. Here $q$ is the persistence length, $M_{\mathrm{L}}$ is the molecular weight per unit length of the wormlike cylinder, and $d$ is the its diameter. The estimated parameter values were found to reproduce quite closely the observed molecular weight dependence

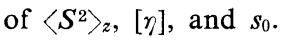

KEY WORDS Poly(phthaloyl-trans-2,5-dimethylpiperazine) / Wormlike Chain / Persistence Length / Dilute Solution / RefractiveIndex Increment / Light Scattering / Intrinsic Viscosity / Sedimentation Coefficient / Yamakawa-Fujii Theory / Benoit-Doty Theory /

Poly (phthaloy1-trans - 2, 5 -dimethylpiperazine) (abbreviated PPDP in the following presentation) is one of a series of polyamides synthesized by Morgan, et al. ${ }^{1}$ The chemical structure of its repeat unit is depicted in Figure 1. The amide bond $\mathrm{CO}-\mathrm{N}$ may be planar and the lateral methyl groups of the piperazine ring may hinder free rotation of the repeat unit about the $\mathrm{C}-\phi$ bond. The PPDP chain should thus undergo a considerably restricted internal rotation and display dilute solution features deviating from the characteristics of typical flexible polymers such as polyisobutylene and polystyrene. The present paper's object is to confirm this prediction. The experiment consisted of preparing a number of well-fractionated samples of PPDP and measuring their molecular weights, radii of gyration, intrinsic viscosities, and sedimentation coefficients in

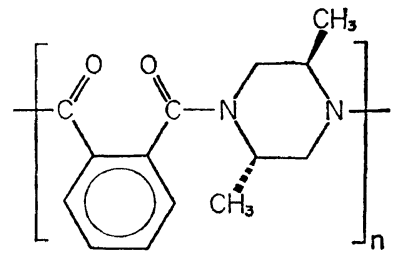

Figure 1. Repeat unit of the PPDP chain.

various organic solvents by using established techniques. The results showed features predictable for semiflexible or stiff chains, and their quantitative interpretation was attempted in terms of the theories for unperturbed wormlike chains.

\section{EXPERIMENTAL}

\section{Samples}

Samples of PPDP were prepared from phthaloyl 
Table I. Polymerization of phthaloyl chloride with trans-2,5-dimethylpiperazine

\begin{tabular}{cccc}
\hline Sample code & {$[\eta], \mathrm{d} l / \mathrm{g}^{\mathrm{d}}$} & Yield, \% & Method \\
\hline 1 & 0.782 & - & $\mathrm{a}$ \\
2 & 1.05 & 81.0 & $\mathrm{a}$ \\
3 & 1.20 & 77.5 & $\mathrm{a}$ \\
4 & 1.55 & 67.1 & $\mathrm{a}$ \\
5 & 0.553 & 91.6 & $\mathrm{~b}$ \\
6 & 0.554 & 72.6 & $\mathrm{c}$ \\
7 & 0.686 & 64.2 & $\mathrm{c}$ \\
\hline
\end{tabular}

a Katz. ${ }^{3}$

b Hodnet and Holmer. ${ }^{4}$

c Morgen and Kwolek. ${ }^{2}$

d Measured in $m$-cresol at $25^{\circ} \mathrm{C}$.

chloride and trans-2,5-dimethylpiperazine. The phthaloyl chloride used was distilled under reduced pressure in a stream of nitrogen gas. Commercially available trans-2,5-dimethylpiperazine was slightly yellowish, and it was used after having been recrystallized once from ethyl acetate and twice from acetone. The colorless plates obtained melted in a range $117.5-117.8^{\circ} \mathrm{C}$ (lit. ${ }^{2} \mathrm{mp} 118^{\circ} \mathrm{C}$ ). The polymerization was carried out by interfacial polycondensation, following the method of $\mathrm{Katz}^{3}$ and that of Hodnet and Holmer, ${ }^{4}$ and also by low-temperature solution polycondensation, according to the method of Morgan and Kwolek. ${ }^{2}$ A typical process using Katz's method was as follows.

Twenty millilitter of $0.4 \%$ aqueous sodium hydroxide, $20 \mathrm{ml}$ of $5-\%$ aqueous sodium lauryl sulfate, $6.5 \mathrm{~g}(0.057 \mathrm{~mol})$ of trans-2,5-dimethylpiperazine, $150 \mathrm{~m} l$ of water, and $25 \mathrm{ml}$ of ethanolfree chloroform were poured into a flask cooled in an ice bath, and $7.2 \mathrm{~m} l(0.050 \mathrm{~mol})$ of phthaloyl chloride dissolved in $25 \mathrm{ml}$ of ethanol-free chloroform was added all at once to this mixture with vigorous stirring. After five minutes, the chloroform was removed by a rotary vacuum evaporator to isolate the polymer formed. The isolated material was washed successively with boiling water, ethanol, and acetone, and then dried in a vacuum oven.

Table I shows the intrinsic viscosities (in $m$-cresol at $25^{\circ} \mathrm{C}$ ) and yields of seven PPDP samples prepared by the methods mentioned.

\section{Fractionation}

PPDP was found to dissolve well in mixtures of methanol and dichloromethane (DCM) of appropriate composition, e.g., that containing 15 vol $\%$ of methanol, although it was not soluble in pure methanol and only partly soluble in pure DCM. Each of the seven samples listed in Table I was subjected to fractional precipitation with DCM containing 15 vol $\%$ of methanol as a solvent and $n$-hexane as a precipitant, and 5 fractions (f-series) were obtained from Sample 1, 11 fractions (F-series) from Samples 2 and 3, 14 fractions (A-series) from Sample 4, 9 fractions (B-series) from Sample 5, and 9 fractions (C-series) from Samples 6 and 7. Appropriate fractions were selected from these and used for the present physical measurements.

\section{Light-Scattering Photometry}

Measurement of Scattering Intensities. Light scattering from PPDP in two solvents, $N$-methyl2-pyrrolidone (NMP) and chloroform, at $25^{\circ} \mathrm{C}$ was investigated by use of a Fica 50 automatic light-scattering photometer in the angular range from 30 to $150^{\circ}$, with vertically polarized light of $436 \mathrm{~nm}$ in wavelength. The procedures of measurement used were the same as described elsewhere. ${ }^{5}$ The absolute Rayleigh ratio for benzene at $25^{\circ} \mathrm{C}$ and $436 \mathrm{~nm}$ was taken to be $46.5 \times 10^{-6} \mathrm{~cm}^{-1}$ for the calibration of the apparatus. ${ }^{6}$ The depolarization, $\rho_{u}$, of benzene was found at be 0.429 at $436 \mathrm{~nm}$, in good agreement with the reported values. ${ }^{\text {? }}$

Polymer solutions and solvents were made free from dust and microgels by centrifugation at $4.9 \times 10^{4}$ gravities for $2 \mathrm{hr}$ in a Sorvall Type RC2-B centrifuge and then directly placed in the light-scattering cell with carefully cleaned pipettes. No filtering was made.

The observed intensities of scattered light as a function of polymer mass concentration $c$ and scattering angle $\theta$ were analyzed by means of Berry's square-root plot $^{8}$ to evaluate $\bar{M}_{w}$ (weightaverage molecular weight), $\left\langle S^{2}\right\rangle_{z} \quad(z$-average mean-square radius of gyration) and $A_{2}$ (second virial coefficient).

Measurements of Specific Refractive Index Increments. The specific refractive index increments of PPDP in various solvents including NMP and chloroform for the wavelengths of 436 and $546 \mathrm{~nm}$ were determined by a differential refractometer of the Schulz-Cantow type, all 
Table II. Refractive index increments, buoyancy factors, and partial specific volumes for PPDP in various solvents at $25^{\circ} \mathrm{C}$

\begin{tabular}{|c|c|c|c|c|c|c|}
\hline \multirow{2}{*}{ Solvent } & \multicolumn{2}{|c|}{$(\partial n / \partial c)_{0}, \mathrm{~cm}^{3} / \mathrm{g}$} & \multirow{2}{*}{$1-\bar{v} \rho_{0}$} & \multirow{2}{*}{$\begin{array}{c}\bar{v} \\
\mathrm{~cm}^{3} / \mathrm{g}\end{array}$} & \multirow{2}{*}{$\begin{array}{c}\rho_{0}, \\
\mathrm{~g} / \mathrm{cm}^{3}\end{array}$} & \multirow{2}{*}{$\begin{array}{c}\eta_{0}, \\
\text { poises }\end{array}$} \\
\hline & $436 \mathrm{~nm}$ & $546 \mathrm{~nm}$ & & & & \\
\hline Hexafluoro-2-propanol & 0.290 & 0.278 & -0.177 & 0.733 & 1.6053 & 一 \\
\hline Chloroform & 0.160 & 0.150 & -0.126 & 0.761 & 1.4801 & - \\
\hline Acetic acid (glacial) & 0.212 & 0.200 & 0.207 & 0.759 & 1.0449 & - \\
\hline$N$-Methyl-2-pyrrolidone & 0.130 & 0.121 & 0.199 & 0.799 & 1.0279 & $1.68_{1}$ \\
\hline$N, N$-Dimethylacetamide & 0.152 & 0.146 & 0.278 & 0.771 & $0.9364_{2}$ & 0.945 \\
\hline Epichlorhydrin & - & - & $0.085_{1}$ & 0.779 & 1.1745 & - \\
\hline$m$-Cresol & $0.065_{6}$ & $0.063_{8}$ & - & - & 1.0300 & - \\
\hline
\end{tabular}

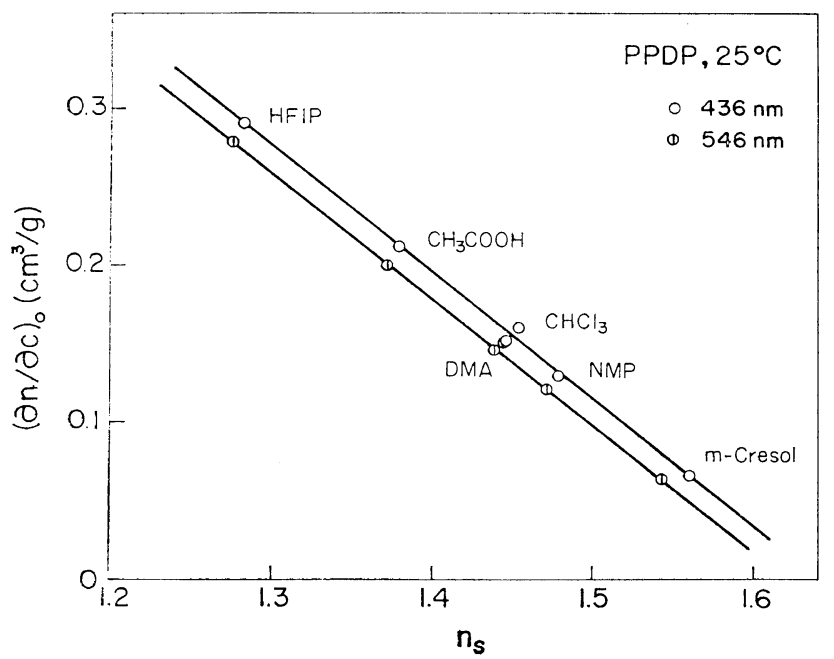

Figure 2. Dependence of refractive index increment $(\partial n / \partial c)_{0}$ on refractive index of solvent $n_{\mathrm{s}}$.

at $25^{\circ} \mathrm{C}$. The apparatus was calibrated with aqueous potassium chloride. The numerical values obtained are given in Table II. Figure 2 shows these values plotted against the refractive indexes of respective solvents. The data points at either wavelength follow a straight line and thus satisfy the Gradstone-Dale relationship

$$
(\partial n / \partial c)_{0}=\bar{v}_{\mathrm{p}}\left(n_{\mathrm{p}}-n_{\mathrm{s}}\right)
$$

where $\bar{v}_{\mathrm{p}}$ is the specific volume of the polymer in the undiluted state and $n_{\mathrm{p}}$ and $n_{\mathrm{s}}$ are the refractive indexes of the pure polymer and the pure solvent, respectively.

\section{Viscometry}

Viscosity measurements were made in $m$-cresol, hexafluoro-2-propanol (HFIP), chloroform, acetic acid, NMP, $N, N$-dimethylacetamide (DMA), and epichlorhydrin (EPCH) at $25^{\circ} \mathrm{C}$ by use of capillary viscometers of the Ubbelohde suspendedlevel type. The viscometer for each solvent was chosen in such a way as to preclude corrections needed for kinetic energy and shearrate dependence. For each pair of polymer fraction and solvent, the flow times were determined at three or more polymer concentrations, and the required intrinsic viscosity was estimated by treating the data with the Huggins plot $^{9}$ as well as the Mead-Fuoss plot. ${ }^{10}$

\section{Ultracentrifugation}

Sedimentation Equilibrium. Seven fractions of PPDP with relatively low molecular weights 


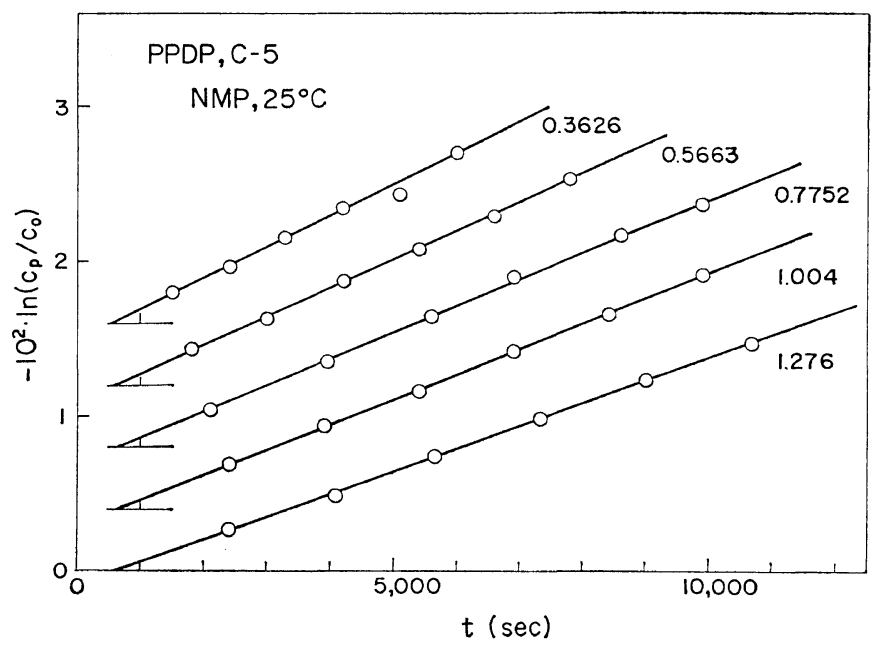

Figure 3. Typical plots of $\ln \left(c_{\mathrm{p}} / c_{0}\right) v s$. $t$ for fraction $\mathrm{C}-5$ in NMP at $25^{\circ} \mathrm{C}$. The numbers attached to the lines indicate the initial concentrations measured in $\mathrm{g} / \mathrm{d} l$.

were investigated by the sedimentation equilibrium method, with NMP at $25^{\circ} \mathrm{C}$ as solvent. The measurements were made in a BeckmanSpinco Model E ultracentrifuge equipped with an electronic speed control system. A Kel-F $12-\mathrm{mm}$ double sector cell was used. The liquid column was adjusted to length from 1.5 to $2.5 \mathrm{~mm}$, and the rotor speeds were chosen so that the ratio $c_{\mathrm{b}} / c_{\mathrm{m}}$ became about three, where $c_{\mathrm{m}}$ and $c_{\mathrm{b}}$ denote the equilibrium solute concentrations at the meniscus and the bottom of the liquid column. The Rayleigh fringe patterns photographed (on the Kodak spectroscopic plates) were read on a Nikon Shadowgraph Model 6 and analyzed by the method of Williams, et al., ${ }^{11}$ to evaluate $\bar{M}_{w}$ and $A_{2}$.

Sedimentation Velocity. Sedimentation coefficients $s$ of PPDP fractions in NMP and DMA at $25^{\circ} \mathrm{C}$ were determined by running a BeckmanSpinco Model E ultracentrifuge. The conventional peak method $^{12}$ was used when freely sedimenting curves were observed. Otherwise use was made of the Baldwin method, ${ }^{13}$ in which the value of $s$ for a given initial concentration $c_{0}$ is determined from the slope (correctly, the initial slope) of a plot of $\ln \left(c_{\mathrm{p}} / c_{0}\right)$ vs. $t$. Here $c_{\mathrm{p}}$ denotes the solute concentration in the plateau region of a schlieren sedimentation curve and $t$ is the time of centrifugation. In order to evaluate $c_{\mathrm{p}}$, each observed schlieren pattern was divided into 20 equal parts from the meniscus to an appropriate position in the plateau region, the heights at these divided points were read (on a Nikon Shadowgraph Model 6), and these were converted to the concentration gradients $\mathrm{d} c / \mathrm{d} r$ in the usual manner. The required $c_{\mathrm{p}}$ was computed by numerical integration of the quantity $(\mathrm{d} c / \mathrm{d} r)\left(r^{2}-r_{m}{ }^{2}\right)$ from the meniscus to the plateau region with the aid of Simpson's one-third rule. Figure 3 illustrates typical plots of $\ln \left(c_{\mathrm{p}} / c_{0}\right)$ vs. $t$ obtained for various initial concentrations of fraction C-5 in NMP at $25^{\circ} \mathrm{C}$.

Partial Specific Volume. The densities of polymer solution and solvent at $25^{\circ} \mathrm{C}$ were measured by use of a Lipkin-Davison pycnometer of $30-\mathrm{m} l$ capacity. The buoyancy factor $1-\bar{v} \rho_{0}$ was estimated from the slope of a plot of solution density $\rho$ vs. polymer concentration $c$, which was strictly linear in the range of the low concentrations studied. The values of $1-\bar{v} \rho_{0}$, together with those of the partial specific volume of the polymer, $\bar{v}$, computed from them with the measured solvent densities $\rho_{0}$, are given in the fourth and fifth column of Table II. The buoyancy factors for HFIP and chloroform are negative, which implies that flotation rather than sedimentation takes place when PPDP is centrifuged in these solvents. 


\section{RESULTS}

Molecular Weight and Second Virial Coefficient Figure 4 illustrates plots of $\left(K c / R_{0}\right)^{1 / 2}$ vs. $c$ for PPDP fractions in NMP at $25^{\circ} \mathrm{C}$, where $K$ is the conventional light-scattering factor and $R_{0}$ is the reduced intensity of scattered light extrapolated to zero of scattering angle. The data points for each fraction are closely fitted by a straight line and allow an accurate evaluation of $\bar{M}_{w}$ and $A_{2}$. Similar data were obtained for PPDP fractions in chloroform at $25^{\circ} \mathrm{C}$. The values of $\bar{M}_{w}$ and $A_{2}$ determined from these light-scattering data are summarized in Table III. The expected agreement of $\bar{M}_{w}$ from experiments with different solvents is satisfactory. Table III also lists the values for $\bar{M}_{w}$ and $A_{2}$ obtained from sedimentation equilibrium measurements on NMP solutions at $25^{\circ} \mathrm{C}$. The values of $A_{2}$ for the two lowest molecular weight fractions C-7 and C-8 appear too large when compared with those of other fractions. At any rate, it is important to observe that, over the range of molecular weights studied, the $A_{2}$ values for either NMP or chloroform solutions of PPDP are comparable to or even larger than those for flexible polymers in good solvents. ${ }^{14}$

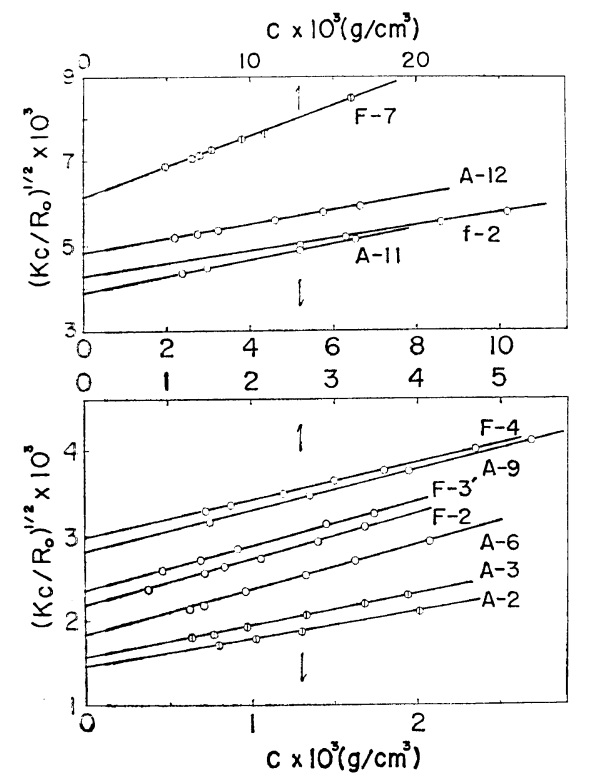

Figure 4. Concentration dependence of $\left(K c / R_{0}\right)^{1 / 2}$ for PPDP fractions in NMP at $25^{\circ} \mathrm{C}$.
Table III. Numerical results from light-scattering and sedimentation equilibrium measurements on PPDP fractions

\begin{tabular}{|c|c|c|c|c|}
\hline $\begin{array}{l}\text { Frac- } \\
\text { tion }\end{array}$ & $\begin{array}{l}\text { Solvent, } \\
25^{\circ} \mathrm{C}\end{array}$ & $\bar{M}_{w} \times 10^{-4}$ & $\begin{array}{c}A_{2} \times 10^{4}, \\
\mathrm{~cm}^{3} \mathrm{~mol} / \mathrm{g}^{2}\end{array}$ & $\left\langle S^{2}\right\rangle_{2}^{1 / 2}$ \\
\hline \multirow[t]{2}{*}{ A- 2} & NMP & 48.2 & 4.78 & 412 \\
\hline & $\mathrm{CHCl}_{3}$ & 48.2 & 6.66 & 433 \\
\hline A- 3 & NMP & 40.6 & 5.84 & 373 \\
\hline F- 1 & $\mathrm{CHCl}_{3}$ & 40.6 & 7.69 & 404 \\
\hline \multirow[t]{2}{*}{ A- 6} & NMP & 29.3 & 5.19 & 313 \\
\hline & $\mathrm{CHCl}_{3}$ & 27.7 & 7.80 & 323 \\
\hline \multirow[t]{2}{*}{ F- 2} & NMP & 21.2 & 5.97 & 270 \\
\hline & $\mathrm{CHCl}_{3}$ & 20.9 & 6.57 & 279 \\
\hline F- $3^{\prime}$ & NMP & 18.3 & 6.32 & 246 \\
\hline A- 8 & $\mathrm{CHCl}_{3}$ & 16.5 & 8.22 & 244 \\
\hline F- 3 & $\mathrm{CHCl}_{3}$ & 16.0 & 9.83 & 240 \\
\hline \multirow[t]{2}{*}{ A- 9} & NMP & 12.8 & 6.83 & 206 \\
\hline & $\mathrm{CHCl}_{3}$ & 12.4 & 8.46 & 211 \\
\hline A-10 & $\mathrm{CHCl}_{3}$ & 10.5 & 9.24 & 194 \\
\hline \multirow[t]{2}{*}{ F- 4} & NMP & 9.35 & 5.58 & 178 \\
\hline & $\mathrm{NMP}^{\mathrm{a}}$ & 9.35 & 8.57 & - \\
\hline A-11 & NMP & 6.65 & 8.43 & 148 \\
\hline$f-2$ & NMP & 5.47 & 6.53 & - \\
\hline F- 6 & $\mathrm{NMP}^{\mathrm{a}}$ & 4.46 & 8.57 & - \\
\hline A-12 & NMP & 4.30 & 7.95 & - \\
\hline B- 4 & $N_{M} P^{a}$ & 3.08 & 9.77 & - \\
\hline F- 7 & NMP & 2.68 & 9.01 & - \\
\hline C- 4 & $\mathrm{NMP}^{\mathrm{a}}$ & 2.02 & 11.3 & - \\
\hline C- 5 & $\mathrm{NMP}^{\mathrm{a}}$ & 1.31 & 10.1 & - \\
\hline C- 7 & $\mathrm{NMP}^{\mathrm{a}}$ & 0.820 & 20.0 & - \\
\hline C- 8 & $\mathrm{NMP}^{\mathrm{a}}$ & 0.602 & 82.5 & - \\
\hline
\end{tabular}

a Determined by sedimentation equilibrium.

\section{Mean-Square Radius of Gyration}

Figure 5 shows plots of $\left(\bar{M}_{w} K c / R_{\theta}\right)_{c=0}^{1 / 2} v s$. $\sin ^{2}(\theta / 2)$ for PPDP fractions in NMP (panel a) and in chloroform (panel b) at $25^{\circ} \mathrm{C}$, where $R_{\theta}$ denotes the reduced intensity of scattered light at a scattering angle $\theta$. The values of $\left\langle S^{2}\right\rangle_{z}^{1 / 2}$ estimated from the slopes of the indicated straight lines are found in the fifth column of Table III. These values are double-logarithmically plotted against $\bar{M}_{w}$ in Figure 6 . The plotted points for either NMP or chloroform can be fitted by a straight line, yielding

$$
\begin{array}{ll}
\left\langle S^{2}\right\rangle_{z}{ }^{1 / 2}=5.8_{0} \times 10^{-9} \bar{M}_{w}{ }^{0.50} & \text { (NMP) } \\
\left\langle S^{2}\right\rangle_{z}{ }^{1 / 2}=4.5_{0} \times 10^{-9} \bar{M}_{w}{ }^{0.53} & \text { (chloroform) }
\end{array}
$$

where $\left\langle S^{2}\right\rangle_{z}{ }^{1 / 2}$ is measured inc entimeters.

The exponents in these equations are equal 


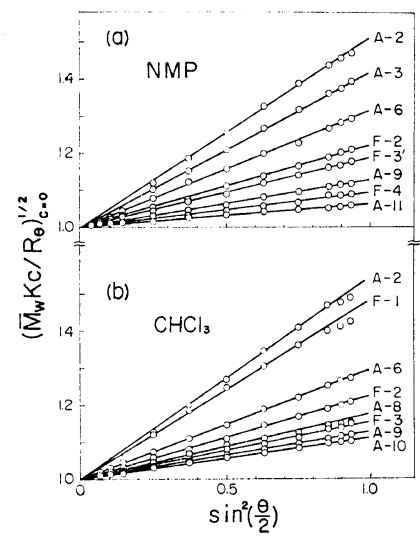

Figure 5. Angular dependence of $\left(\bar{M}_{w} K c / R_{\theta}\right)_{c=0}^{1 / 2}$ for PPDP fractions in NMP (a) and chloroform (b) at $25^{\circ} \mathrm{C}$.

or comparable to those for flexible polymers in theta solvents. ${ }^{14}$ Hence, as far as the molecular weight dependence of $\left\langle S^{2}\right\rangle_{z}$ is concerned, it appears that the molecular chain of PPDP in NMP or in chloroform is in the unperturbed coiled state. However, the data for $A_{2}$ suggest
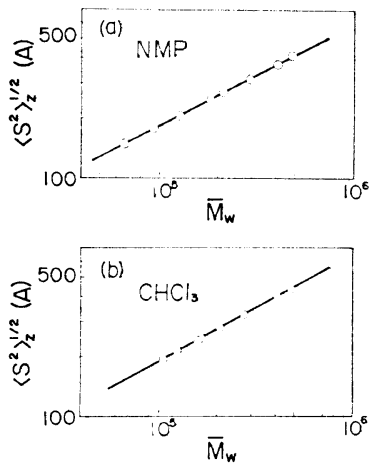

Figure 6. Molecular weight dependence of $\left\langle S^{2}\right\rangle_{2}{ }^{1 / 2}$ for PPDP fractions in NMP (a) and chloroform (b) at $25^{\circ} \mathrm{C}$.

that this is not definitely the case. A discrepancy of a similar nature can be seen in the reported data on polymers whose chains are considered to be stiff or semiflexible. A most distinctive example is cellulose nitrate in acetone. ${ }^{15,16}$ Thus it seems quite legitimate to take the numerical results of Table III as evidence

Table IV. Numerical results from viscosity measurements on PPDP fractions in seven solvents at $25^{\circ} \mathrm{C}$

\begin{tabular}{|c|c|c|c|c|c|c|c|}
\hline \multirow{2}{*}{ Fraction - } & \multicolumn{7}{|c|}{$[\eta], \mathrm{d} l / \mathrm{g}$} \\
\hline & NMP & $\mathrm{CHCl}_{3}$ & $m$-Cresol & HFIP & DMA & $\mathrm{CH}_{3} \mathrm{COOH}$ & $\mathrm{EPCH}$ \\
\hline f- 2 & 0.762 & 0.959 & 1.11 & 1.09 & 0.674 & - & - \\
\hline F- 1 & 2.95 & 3.64 & 4.33 & 4.00 & 2.44 & 2.99 & 2.31 \\
\hline F- 2 & 1.92 & 2.30 & - & - & - & - & - \\
\hline F- $3^{\prime}$ & 1.64 & 2.17 & - & - & - & - & - \\
\hline F- 3 & 1.60 & 一 & 2.21 & - & 1.35 & 1.69 & 1.24 \\
\hline F- 4 & 1.07 & - & 1.61 & 1.53 & 0.962 & - & - \\
\hline F- 6 & 0.675 & - & 0.997 & - & 0.592 & - & -_ \\
\hline F- 7 & 0.467 & 0.570 & 0.712 & - & 0.413 & - & - \\
\hline A- 2 & 3.07 & 4.03 & - & 4.27 & - & 3.12 & - \\
\hline A- 3 & 2.93 & - & - & - & - & - & - \\
\hline A- 6 & 2.30 & 2.90 & 3.49 & - & - & - & - \\
\hline A- 8 & 1.66 & 2.05 & - & 2.26 & - & - & 1.22 \\
\hline A- 9 & 1.34 & 1.65 & - & - & 1.15 & 1.37 & - \\
\hline A-10 & 1.18 & 1.46 & 1.73 & - & 1.03 & - & 0.950 \\
\hline A-11 & 0.864 & 1.06 & - & - & 0.762 & 0.899 & 0.700 \\
\hline A-12 & 0.624 & 0.760 & - & - & 0.530 & - & - \\
\hline B- 4 & 0.520 & 0.617 & 0.726 & 0.695 & 0.422 & 0.544 & 0.420 \\
\hline C- 4 & 0.398 & $(0.460)$ & 0.556 & 0.530 & 0.348 & - & 0.325 \\
\hline C- 5 & 0.276 & $(0.344)$ & 0.390 & - & 0.252 & - & $(0.231)$ \\
\hline C- 7 & $(0.172)$ & $(0.280)$ & $(0.260)$ & $(0.241)$ & $(0.165)$ & - & $(0.150)$ \\
\hline C- 8 & $(0.123)$ & $(0.232)$ & $(0.178)$ & - & - & - & - \\
\hline
\end{tabular}




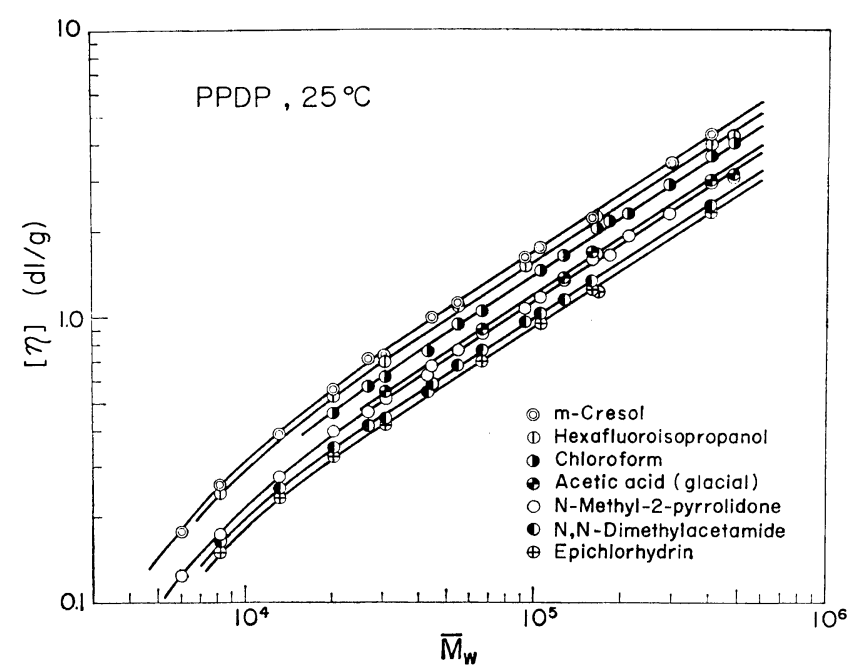

Figure 7. Molecular weight dependence of intrinsic viscosity $[\eta]$ for PPDP fractions in seven solvents at $25^{\circ} \mathrm{C}$.

for a semiflexible nature of the PPDP chain in dilute solution.

\section{Intrinsic Viscosity}

The results from viscosity measurements are summarized in Table IV. Figure 7 illustrates on a log-log graph the molecular weight dependence of $[\eta]$ in seven solvents at $25^{\circ} \mathrm{C}$. The data points for each solvent follow a smooth curve which is convex upward in the range of $\bar{M}_{w}$ below $4 \times 10^{4}$ and tends to be linear for the higher molecular weights. A feature of interest is that the slope of this linear region is essentially independent of the kind of solvent. It is actually close to 0.67 . Thus one can write for PPDP fractions (with $\bar{M}_{w}$ higher than $4 \times 10^{4}$ ) in the seven solvents studied

$$
[\eta]=K \times \bar{M}_{w}^{0.67} \quad([\eta] \text { in } \mathrm{d} l / g)
$$

where $K$ is a constant varying with solvent; for instance, with NMP, the value is $5.2_{2} \times 10^{-4}$. This characteristic dependence of $[\eta]$ on molecular weight and solvent species does not seem explainable in terms of the theory of flexible polymers subject to excluded-volume effects. In fact, such a theory predicts that the front factor and exponent of the Houwink-Mark-Sakurada relation for a given polymer should, in general, vary concomitantly with solvent species. ${ }^{14}$ Thus the viscosity data obtained may be taken as another evidence for a semiflexible nature of the PPDP molecule.

The values of the Huggins constant $k^{\prime}$ were normal $(0.3-0.5)$ for higher molecular weight fractions in any of the seven solvents, but these values in certain solvents tended to increase with decreasing molecular weight. The [ $\eta]$ values for systems with $k^{\prime}$ considered anomalous are given in parentheses in Table IV.

\section{Sedimentation Coefficient}

Reciprocal sedimentation coefficients for PPDP fractions in DMA at $25^{\circ} \mathrm{C}$ are plotted against $c_{0}$ (initial concentration for each sedimentation run) in Figure 8. The data points for each fraction follow a linear relation represented by ${ }^{12}$

$$
s^{-1}=s_{0}^{-1}\left(1+k_{\mathrm{s}} c_{0}\right)
$$

It is to be observed that two sets of plotted points, one from the peak method and the other from the Baldwin method, fall on a single line, as should be expected. Table $\mathrm{V}$ summarizes the values of $s_{0}$ and $k_{\mathrm{s}}$ for all the fractions that were subjected to sedimentation velocity experiments. These data for $s_{0}$ are quite closely represented by

$$
\begin{array}{ll}
s_{0}=1.2_{9} \times 10^{-15} \bar{M}_{w}^{0.38} & (\mathrm{NMP}) \\
s_{0}=2.9_{7} \times 10^{-15} \bar{M}_{w}^{0.38} & (\mathrm{DMA})
\end{array}
$$

where $s_{0}$ is measured in seconds. Again it should be noted that the exponents to $\bar{M}_{w}$ for NMP and DMA solutions are equal. Though 
not shown graphically here, there is a very close correlation between $s_{0}$ and $k_{\mathrm{s}}$ for each of these solutions.

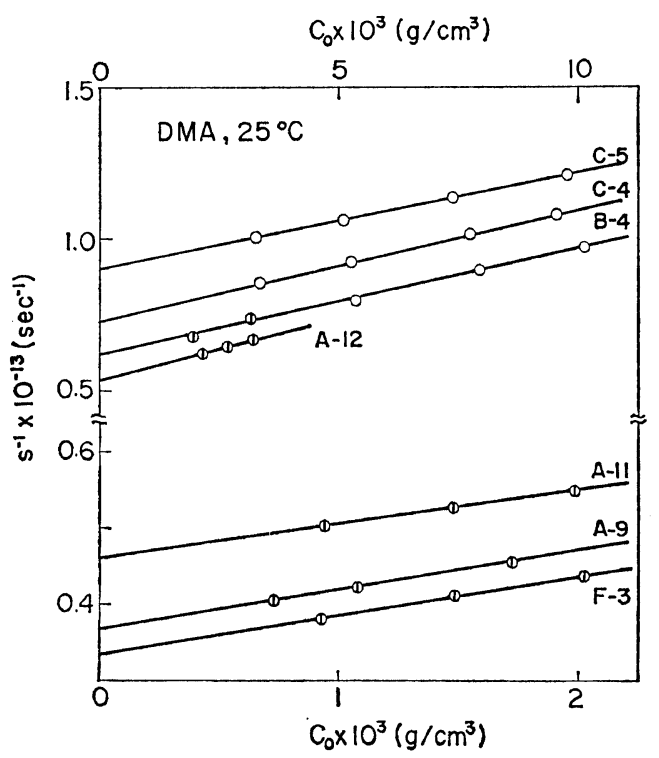

Figure 8. Concentration dependence of sedimentation coefficient $s$ for PPDP fractions in DMA at $25^{\circ} \mathrm{C}$ : (D) data from the peak method; ( $\left.\bigcirc\right)$ data from the Baldwin method.

Table V. Numerical results from sedimentation velocity measurements on PPDP fractions in NMP and DMA at $25^{\circ} \mathrm{C}$

\begin{tabular}{clll}
\hline Fraction & Solvent & $\begin{array}{c}s_{0} \times 10^{13}, \\
\text { sec }\end{array}$ & $\begin{array}{c}k_{\mathrm{s}}, \\
\mathrm{d} l / \mathrm{g}\end{array}$ \\
\hline F- 1 & NMP $^{\mathrm{a}}$ & 1.79 & 4.46 \\
A- 6 & NMP $^{\mathrm{a}}$ & 1.47 & 3.20 \\
F- 2 & NMP $^{\mathrm{a}}$ & 1.32 & 2.70 \\
F- 3 & NMP $^{\mathrm{a}}$ & 1.20 & 2.29 \\
& DMA $^{\mathrm{a}}$ & 2.99 & 1.52 \\
A- 9 & DMA $^{\mathrm{a}}$ & 2.73 & 1.42 \\
F- 4 & NMP $^{\mathrm{b}}$ & 1.04 & 1.55 \\
A-11 & DMA $^{\mathrm{a}}$ & 2.18 & 0.980 \\
f- 2 & NMP $^{\mathrm{b}}$ & 0.813 & 1.06 \\
A-12 & DMA $^{\mathrm{a}}$ & 1.85 & 0.719 \\
B- 4 & NMP $^{\mathrm{c}}$ & 0.704 & 0.872 \\
& DMA $^{\mathrm{b}}$ & 1.61 & 0.565 \\
C- 4 & DMA $^{\mathrm{c}}$ & 1.36 & 0.489 \\
C- 5 & NMP $^{\mathrm{c}}$ & 0.488 & 0.523 \\
& DMA $^{\mathrm{c}}$ & 1.11 & 0.355
\end{tabular}

a The peak method.

b The peak method and the Baldwin method.

c The Baldwin method.

\section{DISCUSSION}

The semiflexible nature of the PPDP chain apparent from the experimental data presented above may be ascribed to a steric hindrance of internal rotation of each repeat unit about the $\mathrm{C}-\phi$ bond. In order to visualize the stiffness of the chain, we attempted analysis of the data in terms of the unperturbed wormlike chain model, which is a representative model for stiff chains. ${ }^{14,17}$ The results of this analysis are presented below.

There are now available for wormlike chains the Benoit-Doty expression ${ }^{18}$ for $\left\langle S^{2}\right\rangle$ and the Yamakawa-Fujii expressions ${ }^{19,20}$ for $[\eta]$ and $s_{0}$. Three quantities $q, M_{\mathrm{L}}$, and $d$ characterize these expressions, where $q$ is the persistence length, $M_{\mathrm{L}}$ is the molecular weight per unit length of the wormlike cylinder, and $d$ is its diameter. Yamakawa and Fujii have proposed a method for evaluating the three parameters from a combination of data for [ $\eta]$ and $s_{0}$ as functions of molecular weight $M$ and have applied this successfully to typical stiff chains such as DNA, cellulose nitrate, and poly ( $n$-butyl isocyanate). ${ }^{20}$ However, as has been pointed out by them, it cannot be used for an unambiguous determination of chain parameters unless the chains are very stiff. In fact we found this to be the case with our PPDP. Hence, in this present analysis, we used a different approach; it is deduced from the Yamakawa-Fujii theory for intrinsic viscosity, combined with the Benoit-Doty expression for mean-square radius of gyration and an assumption for the partial specific volume.

\section{Method of Analysis}

According to the Yamakawa-Fujii theory for unperturbed wormlike chains, the intrinsic viscosity of this kind of chain may be expressed in the region of high molecular weights by ${ }^{20}$

$$
\begin{aligned}
{[\eta]=} & {\left[\Phi_{\infty} /\left(M_{\mathrm{L}} / 2 q\right)^{3 / 2}\right] M^{1 / 2} /\left[1-\left(2 q M_{\mathrm{L}}\right)^{1 / 2}\right.} \\
& \left.\times g(d / 2 q) M^{-1 / 2}+0\left(M^{-1}\right)\right]
\end{aligned}
$$

where $g(d / 2 q)$, denoted by $C_{1}$ in YamakawaFujii's original paper, is a known function of $d / 2 q$, and $\Phi_{\infty}$ is a numerical constant. In fact, $\Phi_{\infty}$ is the limiting value of the Flory viscosity factor $\Phi$ for infinitely large $M$, where $\Phi$ is 
defined by ${ }^{14}$

$$
[\eta]=\Phi\left(6\left\langle S^{2}\right\rangle\right)^{3 / 2} / M
$$

Equation 8 may be put in the form

$$
\begin{aligned}
M^{1 / 2} /[\eta]= & {\left[\left(M_{\mathrm{L}} / 2 q\right)^{3 / 2} / \Phi_{\infty}\right]\left[1-\left(2 q M_{\mathrm{L}}\right)^{1 / 2}\right.} \\
& \left.\times g(d / 2 q) M^{-1 / 2}+0\left(M^{-1}\right)\right]
\end{aligned}
$$

and therefore $\left(M_{\mathrm{L}} / 2 q\right)^{3 / 2} / \Phi_{\infty}$ and $-\left(2 q M_{\mathrm{L}}\right)^{1 / 2} \times$ $g(d / 2 q)$ can be evaluated from the originate intercept and initial slope of a plot of $M^{1 / 2} /[\eta]$ vs. $M^{-1 / 2}$. These two quantities contain four unknown parameters $q, M_{\mathrm{L}}, d$, and $\Phi_{\infty}$, so that two more relations are needed for their unequivocal determination.

Equations 8 through 10 apply to samples which are monodisperse in molecular weight. For such samples we may use a theoretically derived value of $2.87 \times 10^{23}$ for the parameter $\Phi_{\infty},{ }^{20}$ and only one additional relation is need for the evaluation of $q, M_{\mathrm{L}}$, and $d$. However, the polymer fractions used for the present measurement were undoubtedly polydisperse, so that this convention cannot be applied. It is reasonable in the case of such samples to treat $\Phi_{\infty}$ as a parameter to be evaluated from experiment. In addition, as is usually done, $M$ and $\left\langle S^{2}\right\rangle$ in eq $8-10$ may be replaced by $\bar{M}_{w}$ and $\left\langle S^{2}\right\rangle_{z}$, respectively.

The Benoit-Doty expression ${ }^{18}$ for $\left\langle S^{2}\right\rangle$ of unperturbed wormlike chains is

$$
\begin{aligned}
\left\langle S^{2}\right\rangle= & \left(q / 3 M_{\mathrm{L}}\right) M-q^{2}\left\{1-2 q M_{\mathrm{L}} / M\right. \\
& \left.+2\left(q M_{\mathrm{L}} / M\right)^{2}\left[1-\exp \left(-M / q M_{\mathrm{L}}\right)\right]\right\}
\end{aligned}
$$

Substitution of this equation and eq 8 into 9 yields

$$
1 / \Phi=\left(1 / \Phi_{\infty}\right)\left[1-\left(2 q M_{\mathrm{L}}\right)^{1 / 2} g(d / 2 q) M^{-1 / 2}+0\left(M^{-1}\right)\right]
$$

which suggests what plot should be used for the evaluation of $\Phi_{\infty}$ from a series of $\Phi$ values for different molecular weights. That is to say, we may plot $\Phi^{-1}$ computed from observed $\bar{M}_{w}$, $\left\langle S^{2}\right\rangle_{z}$, and $[\eta]$ against $\bar{M}_{w}{ }^{-1 / 2}$ and find the ordinate intercept of the resulting plot by extrapolation. In this case, the value of $-\left(2 q M_{\mathrm{L}}\right)^{1 / 2} g(d / 2 q)$ also may be determined from the initial slope of the plot together with the $\Phi_{\infty}$ value so obtained.

As a necessary fourth relation, we propose, in accordance with Tsuji, et al., ${ }^{5}$ $\bar{v}=\pi N_{\mathrm{A}} d^{2} / 4 M_{\mathrm{L}} \quad\left(N_{\mathrm{A}}=\right.$ Avogadro's number $)$

which is derived from the assumption that the hydrodynamic volume occupied by one gram of the wormlike cylinder is equal to the partial specific volume of the polymer molecule. This assumption seems reasonable for chains which are stiff so that their local shape may be regarded as cylindrical.

Evaluation of $q, M_{\mathrm{L}}$, and $d$ for the PPDP Chain Figure 9 illustrates plots of $\bar{M}_{w}{ }^{1 / 2} /[\eta]$ vs. $\bar{M}_{w}{ }^{-1 / 2}$ derived from our viscosity data on PPDP fractions. Within the range of molecular weights indicated the data points for each of the solvents studied are fitted by a straight line. This means that the third term on the right-hand side of eq 10 is negligibly small for these systems and molecular weights.

Figure 10 shows that the plots of $1 / \Phi$ vs. $\bar{M}_{w}{ }^{-1 / 2}$ for the PPDP fractions in NMP and chloroform are linear in the indicated range of molecular weights and can be extrapolated to an essentially identical intercept, which yields $2.0_{5} \times 10^{23}$ for $\Phi_{\infty}$. This value is about $30 \%$ smaller than the theoretically predicted one for monodisperse samples, but seems reasonable for our PPDP fractions which would have been moderately polydisperse. We assume that this applies for these fractions in the other solvents studied.

We computed three parameters $q, M_{\mathrm{L}}$, and $d$, using the values of $\left(M_{\mathrm{L}} / 2 q\right)^{3 / 2} / \Phi_{\infty}$ and $-\left(2 q M_{\mathrm{L}}\right)^{1 / 2} g(d / 2 q)$ estimated from the intercepts and slopes of the lines in Figure 9,

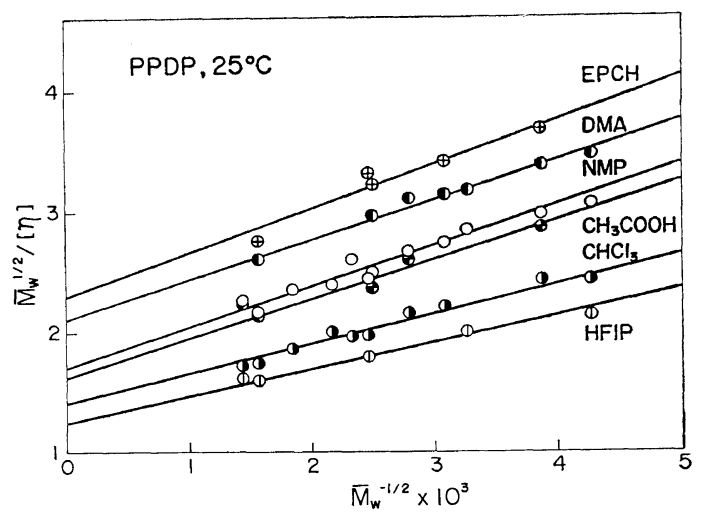

Figure 9. Plots of $\bar{M}_{w}^{1 / 2} /[\eta]$ vs. $\bar{M}_{w}^{-1 / 2}$ for PPDP fractions in six solvents at $25^{\circ} \mathrm{C}$. 


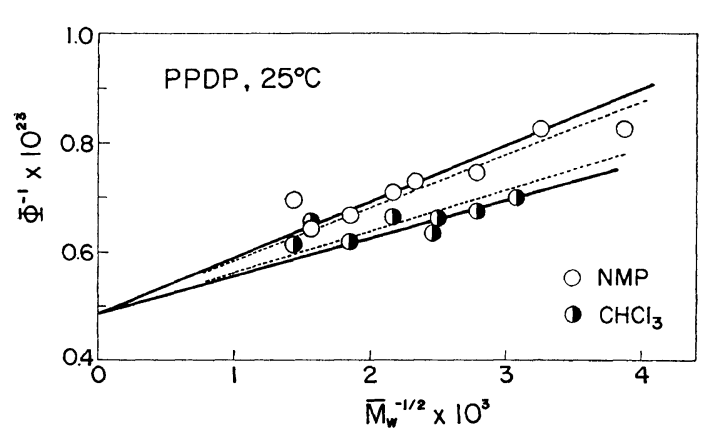

Figure 10. Plots of $\Phi^{-1}$ vs. $\bar{M}_{w}^{-1 / 2}$ for PPDP fractions in NMP and chloroform at $25^{\circ} \mathrm{C}$. Theoretical values (dotted lines) are calculated from the Yamakawa-Fujii theory, ${ }^{20}$ using the values of the parameters listed in Table VI.

Table VI. Wormlike chain parameters of PPDP in various solvents

\begin{tabular}{lcccc}
\hline \multicolumn{1}{c}{ Solvent } & $q, \AA$ & $\begin{array}{c}M_{\mathrm{L}}, \\
\text { daltons } / \AA\end{array}$ & $d, \AA$ & $\Phi_{\infty} \times 10^{-23}$ \\
\hline HFIP & 41 & 33 & 7.1 & $\mathrm{a}$ \\
Chloroform & 37 & 33 & 7.3 & $2.0_{5}$ \\
Acetic acid & 33 & 32 & 7.1 & $\mathrm{a}$ \\
NMP & 33 & 33 & 7.4 & $2.0_{5}$ \\
DMA & 28 & 31 & 7.2 & $\mathrm{a}$ \\
EPCH & 26 & 31 & 7.2 & $\mathrm{a}$ \\
\hline
\end{tabular}

a Assumed $\Phi_{\infty}=2.0_{5} \times 10^{23}$.

$\Phi_{\infty}=2.0_{5} \times 10^{23}$, and the experimental values of $\bar{v}$ (Table II) substituted into eq 13. The resulting values are given in Table VI. Interestingly, the values of $M_{\mathrm{L}}$ and $d$ are essentially independent of the kind of solvent. This indicates that the observed characteristic dependence of $[\eta]$ of PPDP on solvent species is simply ascribable to the variation of $q$ with the solvent, because, according to the Yamakawa-Fujii theory, ${ }^{20}$ the intrinsic viscosity of an unperturbed wormlike chain of given molecular weight is expressed in terms of $q, M_{\mathrm{L}}$, and $d$. The values $31-33$ daltons/A obtained for $M_{\mathrm{L}}$ are close to 30.3 daltons/ $\AA$ which the PPDP chain would assume if it were fully stretched. This similar finding has been reported by Yamakawa and Fujii who analyzed hydrodynamic data on typical stiff chains such as cellulose nitrate and poly( $n$-butyl isocyanate) in terms of their theory of wormlike chains. The diameter values of $7.1-7.4 \AA$ are not unreasonable. Finally, the $q$ values obtained indicate that the PPDP chain, at least in the solvents studied, is only moderately stiff. Certainly, it is far flexible in comparison with poly $n$-butyl isocyanate) for which the values of $q$ are of the order of several hundred Angstroms, but its dilute solution properties still display molecular weight and solvent dependences which are characteristically different from those of typical flexible polymers.

Comparison of Observed Results and Theoretical Predictions

The values of $q, M_{\mathrm{L}}, d$, and $\Phi_{\infty}$ estimated above from the asymptotic behavior of $[\eta]$ and $\Phi$ in conjunction with eq 13 for $\bar{v}$ were substituted into the Benoit-Doty theory for $\left\langle S^{2}\right\rangle$ and the Yamakawa-Fujii theory for $[\eta]$ and the translational friction coefficient $\Xi$ to compute these quantities as functions of $M$. Actually, the numerical tables provided by Professor Yamakawa were used for the computation of $[\eta]$ and $\Xi$.

Figure 11 shows a comparison of the observed values of $\left\langle S^{2}\right\rangle_{z} / \bar{M}_{w}$ (points) for NMP and chloroform solutions with the predictions (solid curves) from eq 11. The agreement between observed and computed values is satisfactory for both NMP and chloroform solutions. However, we recognize that our light-scattering determinations of $\left\langle S^{2}\right\rangle_{z}$ failed to approach the region of $\bar{M}_{w}$ where the sharp fall of $\left\langle S^{2}\right\rangle / M$ characteristic of stiff chains takes place and hence were unable to make a real check of eq 11.

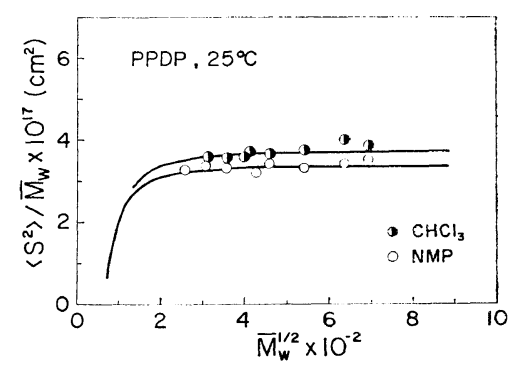

Figure 11. Comparison of the data of $\left\langle S^{2}\right\rangle_{2}$ for PPDP fractions in NMP and chloroform with the theoretical values calculated from eq 11, using the values of the parameters listed in Table VI. 

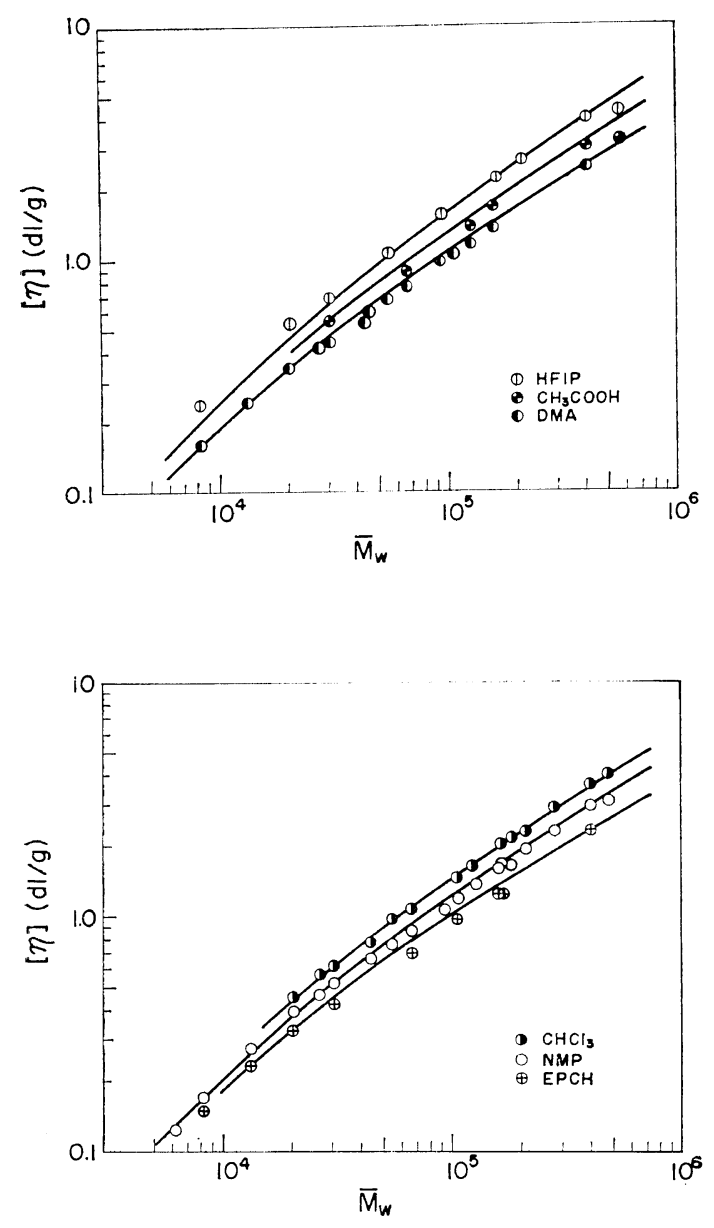

Figure 12. Comparison of the measured [ $\eta]$ (various points) for PPDP fractions in six solvents with the theoretical values (solid lines) calculated from the Yamakawa-Fujii theory, ${ }^{20}$ using the values of the parameters listed in Table VI.

The computed values of $[\eta]$ as a function of molecular weight are compared on a log-log graph with the experimental data in Figure 12. Again we can see a satisfactory agreement between theory and experiment for each of the six solvents indicated. The points to note is that the Yamakawa-Fujii theory, with the parameter values chosen, predicts quite correctly the characteristic downward curvature of the observed $\log [\eta] v s . \log \bar{M}_{w}$ plots. The computed values for $\Phi$ are shown as dotted lines in Figure 10. Taking into account experimental errors, we may say that the experimental values of $\Phi$

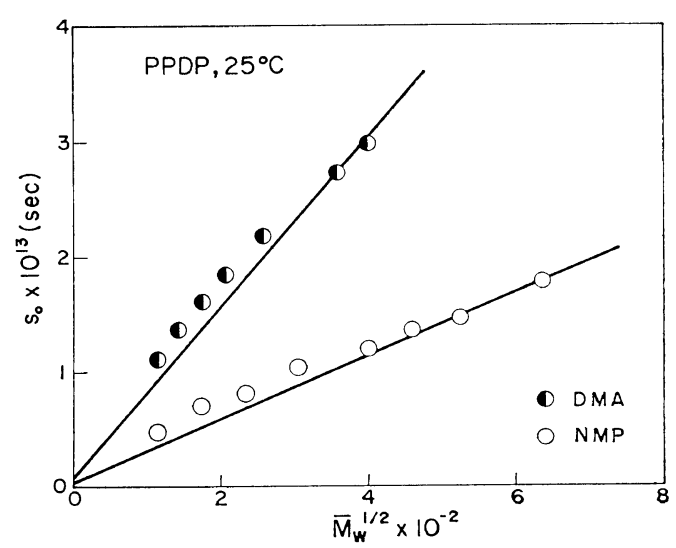

Figure 13. Comparison of the data of $s_{0}$ for PPDP fractions in NMP and DMA with the theoretical values calculated from the Yamakawa-Fujii theory, ${ }^{19}$ using the values of the parameters listed in Table VI.

agree with the computed values. This also support the validity of the parameter values chosen.

Finally, in Figure 13, the computed values of $s_{0}$ plotted against the square root of molecular weight are compared with the observed results for NMP and DMA solutions. The values of $1-\bar{v} \rho_{0}$ and $\eta_{0}$ (viscosity coefficient of pure solvent) used in converting $\Xi$ to $s_{0}$ are given in Table II. Here the agreement between theory and experiment is not as close as was found with intrinsic viscosity, but the observed values of $s_{0}$ are systematically larger than the theoretical ones in the region of $\bar{M}_{w}$ lower than $10^{5}$. The reasons for this are not yet clear to us. We may point out that there is considerable difficulty associated with an accurate determination of small $s_{0}$ as seen in this molecular weight.

In conclusion, the values of parameters $q$, $M_{\mathrm{L}}, d$, and $\Phi_{\infty}$ listed in Table VI can describe fairly well the dimensional and hydrodynamic properties of PPDP in the solvents studied over a wide range of molecular weights. In other words, the PPDP chain in these solvents behaves like a wormlike chain of Kratky and Porod, ${ }^{21}$ and no allowance for excluded-volume effects is needed to interpret its dilute solution behavior.

Acknowledgment. The authors wish to thank Professor H. Yamakawa of the Department of 
Polymer Chemistry, Kyoto University, who kindly provided them with the numerical tables required for the computation of $[\eta]$ and $E$.

\section{REFERENCES}

1. P.W. Morgan, "Condensation Polymers: By Interfacial and Solution Methods," Interscience, New York, N.Y., 1965.

2. P. W. Morgan and S. L. Kwolek, J. Polym. Sci., Part A-2, 181 (1964).

3. M. Katz, ibid., 40, 337 (1959).

4. E. M. Hodnet and D. A. Holmer, ibid., 58, 1415 (1962).

5. T. Tsuji, T. Norisuye, and H. Fujita, Polym. J., 7, 558 (1975).

6. Gj. Deželič and J. Vavra, Croat. Chim. Acta, 38, 35 (1966).

7. J.P. Kratohvil, Gj. Deželič, M. Kerker, and E. Matijevič, J. Polym. Sci., 57, 59 (1962).

8. G. C. Berry, J. Chem. Phys., 444550 (1966).

9. M. L. Huggins, J. Am. Chem. Soc., 64, 2716 (1942).

10. D. F. Mead and R. M. Fuoss, ibid., 64, 277 (1942).
11. J.W. Williams, R. L. Baldwin, K. E. Van Holde, and H. Fujita, Chem. Rev., 58, 715 (1958).

12. H. Fujita, "Foundations of Ultracentrifugal Analysis," Interscience, New York, N.Y., 1975.

13. R. L. Baldwin, Biochem. J., 55, 644 (1953).

14. H. Yamakawa, "Modern Theory of Polymer Solutions," Harper \& Row, New York, N.Y. 1971.

15. G. V. Schulz and E. Penzel, Makromol. Chem., 112, 260 (1968).

16. E. Penzel and G. V. Schulz, ibid., 113, 64 (1968).

17. H. Yamakawa, Pure Appl. Chem., 46, 135 (1976).

18. H. Benoit and P. Doty, J. Phys. Chem., 57, 958 (1953).

19. H. Yamakawa and M. Fujii, Macromolecules, 6, 407 (1973).

20. H. Yamakawa and M. Fujii, ibid., 7, 128 (1974).

21. O. Kratky and G. Porod, Rec. Trav. Chim., 68, 1106 (1949). 